\title{
On the maximum size of connected hypergraphs without a path of given length
}

\author{
Ervin Gyôri * Abhishek Methuku ${ }^{\dagger}$ Nika Salia ${ }^{\ddagger}$ Casey Tompkins ${ }^{\S}$ \\ Máté Vizer 9
}

July 30, 2018

\begin{abstract}
In this note we asymptotically determine the maximum number of hyperedges possible in an $r$-uniform, connected $n$-vertex hypergraph without a Berge path of length $k$, as $n$ and $k$ tend to infinity. We show that, unlike in the graph case, the multiplicative constant is smaller with the assumption of connectivity.
\end{abstract}

\section{Introduction}

Let $P_{k}$ denote a path consisting of $k$ edges in a graph $G$. There are several notions of paths in hypergraphs the most basic of which is due to Berge. A Berge path of length $k$ is a set of $k+1$ distinct vertices $v_{1}, v_{2}, \ldots, v_{k+1}$ and $k$ distinct hyperedges $h_{1}, h_{2}, \ldots, h_{k}$ such that for $1 \leq i \leq k, v_{i}, v_{i+1} \in h_{i}$. A Berge path is also denoted simply as $P_{k}$, and the vertices $v_{i}$ are called basic vertices. If $v_{1}=v$ and $v_{k+1}=w$, then we call the Berge path a Berge $v$-w-path. A hypergraph $\mathcal{H}$ is called connected if for any $v \in V(\mathcal{H})$ and $w \in V(\mathcal{H})$ there is a Berge $v$-w-path. Let $N_{s}(G)$ denote the number of $s$-vertex cliques in the graph $G$.

A classical result of Erdôs and Gallai [3] asserts that

Theorem 1 (Erdôs-Gallai). Let $G$ be a graph on $n$ vertices not containing $P_{k}$ as a subgraph, then

$$
|E(G)| \leq \frac{(k-1) n}{2}
$$

\footnotetext{
${ }^{*}$ Alfréd Rényi Institute of Mathematics, Hungarian Academy of Sciences. e-mail: gyori.ervin@renyi.mta.hu

${ }^{\dagger}$ Central European University, Budapest. e-mail: abhishekmethuku@gmail.com

${ }^{\ddagger}$ Central European University, Budapest. e-mail: Nika_Salia@phd.ceu.edu

$\S$ Alfréd Rényi Institute of Mathematics, Hungarian Academy of Sciences. e-mail: ctompkins496@gmail.com

『Alfréd Rényi Institute of Mathematics, Hungarian Academy of Sciences. e-mail: vizermate@gmail.com.
} 
In fact, Erdôs and Gallai deduced this result as a corollary of the following stronger result about cycles,

Theorem 2 (Erdôs-Gallai). Let $G$ be a graph on $n$ vertices with no cycle of length at least $k$, then

$$
|E(G)| \leq \frac{(k-1)(n-1)}{2}
$$

Kopylov [5] and later Balister, Gyóri, Lehel and Schelp [1] determined the maximum number of edges possible in a connected $P_{k}$-free graph.

Theorem 3. Let $G$ be a connected n-vertex graph with no $P_{k}, n>k \geq 3$. Then $|E(G)|$ is bounded above by

$$
\max \left\{\left(\begin{array}{c}
k-1 \\
2
\end{array}\right)+n-k+1,\left(\begin{array}{c}
\left\lceil\frac{k+1}{2}\right\rceil \\
2
\end{array}\right)+\left\lfloor\frac{k-1}{2}\right\rfloor\left(n-\left\lceil\frac{k+1}{2}\right\rceil\right)\right\} .
$$

Observe that, although the upper bound is lower in the connected case, it is nonetheless the same asymptotically. Balister, Gyóri, Lehel and Schelp also determined the extremal cases.

Definition 1. The graph $H_{n, k, a}$ consists of 3 disjoint vertex sets $A, B, C$ with $|A|=a$, $|B|=n-k+a$ and $|C|=k-2 a$. $H_{n, k, a}$ contains all edges in $A \cup C$ and all edges between $A$ and $B . B$ is taken to be an independent set. The number of s-cliques in this graph is

$$
f_{s}(n, k, a)=\left(\begin{array}{c}
k-a \\
s
\end{array}\right)+(n-k+a)\left(\begin{array}{c}
a \\
s-1
\end{array}\right) .
$$

The upper bound of Theorem 3$]$ is attained for the graph $H_{n, k, 1}$ or $H_{n, k,\left\lfloor\frac{k-1}{2}\right\rfloor}$.

We now mention some recent results of Luo [6] which will be essential in our proof.

Theorem 4 (Luo). Let $n-1 \geq k \geq 4$. Let $G$ be a connected $n$-vertex graph with no $P_{k}$, then the number of s-cliques in $G$ is at most

$$
\max \left\{f_{s}(n, k,\lfloor(k-1) / 2\rfloor), f_{s}(n, k, 1)\right\} .
$$

As a corollary, she also showed

Corollary 1 (Luo). Let $n \geq k \geq 3$. Assume that $G$ is an $n$-vertex graph with no cycle of length $k$ or more, then

$$
N_{s}(G) \leq \frac{n-1}{k-2}\left(\begin{array}{c}
k-1 \\
s
\end{array}\right) .
$$

Győri, Katona and Lemons [4] initiated the study of Berge $P_{k}$-free hypergraphs. They proved 
Theorem 5 (Gyóri-Katona-Lemons). Let $\mathcal{H}$ be an r-uniform hypergraph with no Berge path of length $k$. If $k>r+1>3$, we have

$$
|E(\mathcal{H})| \leq \frac{n}{k}\left(\begin{array}{l}
k \\
r
\end{array}\right)
$$

If $r \geq k>2$, we have

$$
|E(\mathcal{H})| \leq \frac{n(k-1)}{r+1} .
$$

The case when $k=r+1$ was settled later [2]:

Theorem 6 (Davoodi-Győri-Methuku-Tompkins). Let $\mathcal{H}$ be an $n$-vertex $r$-uniform hypergraph. If $|E(\mathcal{H})|>n$, then $\mathcal{H}$ contains a Berge path of length at least $r+1$.

Our main result is the asymptotic upper bound for the connected version of Theorem 5 , as $n$ and $k$ tend to infinity.

Theorem 7. Let $\mathcal{H}_{n, k}$ be a largest $r$-uniform connected n-vertex hypergraph with no Berge path of length $k$, then

$$
\lim _{k \rightarrow \infty} \lim _{n \rightarrow \infty} \frac{\left|E\left(\mathcal{H}_{n, k}\right)\right|}{k^{r-1} n}=\frac{1}{2^{r-1}(r-1) !} .
$$

A construction yielding the bound in Theorem 7 is given by partitioning an $n$-vertex set into two classes $A$, of size $\left\lfloor\frac{k-1}{2}\right\rfloor$, and $B$, of size $n-\left\lfloor\frac{k-1}{2}\right\rfloor$ and taking $X \cup\{y\}$ as a hyperedge for every $(r-1)$-element subset $X$ of $A$ and every element $y \in B$. This hypergraph has no Berge $P_{k}$ as we could have at most $\left\lfloor\frac{k-1}{2}\right\rfloor$ basic vertices in $A$ and $\left\lfloor\frac{k-1}{2}\right\rfloor+1$ basic vertices in $B$, thus yielding less than the required $k+1$ basic vertices.

Observe that in Theorem 5 the corresponding limiting value of the constant factor is $\frac{1}{r !}$ which is $\frac{2^{r-1}}{r}$ times larger than in the connected case. Note that the ideas of the proof of Theorem 7 can be used to prove that the limiting value of the constant factor in Theorem 5 is $\frac{1}{r !}$.

\section{Proof of Theorem 7}

We will use the following simple corollary of Theorem 4,

Corollary 2. Let $G$ be a connected graph on $n$ vertices with no $P_{k}$, then $G$ has at most

$$
\frac{k^{r-1} n}{2^{r-1}(r-1) !}
$$

$r$-cliques if $n \geq c_{k, r}$ for some constant $c_{k, r}$ depending only on $k$ and $r$.

Proof. From Theorem 4, it follows that for large enough $n$, the number of $r$-cliques is at most

$$
\left(n-\left\lfloor\frac{k-1}{2}\right\rfloor\right)\left(\begin{array}{c}
\left\lfloor\frac{k-1}{2}\right\rfloor \\
r-1
\end{array}\right)+\left(\begin{array}{c}
\left\lfloor\frac{k-1}{2}\right\rfloor \\
r
\end{array}\right)+\left(\begin{array}{c}
\left\lfloor\frac{k-1}{2}\right\rfloor \\
r-2
\end{array}\right)<n\left(\begin{array}{c}
\frac{k}{2} \\
r-1
\end{array}\right) .
$$


Given an $r$-uniform hypergraph $\mathcal{H}$ we define the shadow graph of $\mathcal{H}$, denoted $\partial \mathcal{H}$ to be the graph on the same vertex set with edge set:

$$
E(\partial \mathcal{H}):=\{\{x, y\}:\{x, y\} \subset e \in E(\mathcal{H})\}
$$

Definition 2. If $r=3$, then we call an edge $e \in E(\partial \mathcal{H})$ fat if there are at least 2 distinct hyperedges $h_{1}, h_{2}$ with $e \subset h_{1}, h_{2}$. If $r>3$, then we call an edge $e \in E(\partial \mathcal{H})$ fat if there are at least $k$ distinct hyperedges $h_{1}, h_{2}, \ldots, h_{k}$ in $\mathcal{H}$ with $e \subset h_{i}$ for $1 \leq i \leq k$.

We call an edge $e \in E(\partial \mathcal{H})$ thin if it is not fat.

Thus, the set $E(\partial \mathcal{H})$ decomposes into the set of fat edges and the set of thin edges. We will refer to the graph whose edges consist of all fat edges in $\partial \mathcal{H}$ as the fat graph and denote it by $F$.

Lemma 1. There is no $P_{k}$ in the fat graph $F$ of the hypergraph $\mathcal{H}$.

Proof. Suppose we have such a $P_{k}$ with edges $e_{1}, e_{2}, \ldots, e_{k}$. For $r=3$, if a hyperedge contains two edges from the path, then it must contain consecutive edges $e_{i}, e_{i+1}$. Select hyperedges $h_{1}, h_{2}, \ldots, h_{k}$ where $e_{i} \subset h_{i}$ in such a way that $h_{i+1}$ is different from $h_{i}$ for all $1 \leq i \leq k-1$, and these edges yield the required Berge path.

Suppose now that $r>3$, we will find a Berge path of length $k$ in $\mathcal{H}$, greedily. For $e_{1}$, select an arbitrary hyperedge $h_{1}$ containing it. Suppose we have found a distinct hyperedge $h_{i}$ containing the fat edge $e_{i}$ for all $1 \leq i<i^{*}$. Since the edge $e_{i^{*}}$ is fat, there are at least $k$ different hyperedges $h_{i^{*}}^{1}, h_{i^{*}}^{2}, \ldots, h_{i^{*}}^{k}$ containing it. Select one of them, say $h_{i^{*}}^{j}$, which is not equal to any of $h_{1}, h_{2}, \ldots, h_{i^{*}-1}$. Thus, we may find distinct hyperedges $h_{1}, h_{2}, \ldots, h_{k}$ where $e_{i} \subset h_{i}$ for $1 \leq i \leq k$, and thus, we have a Berge path of length $k$.

We call a hyperedge $h \in E(\mathcal{H})$ fat if $h$ contains no thin edge. Let $\mathcal{F}$ denote the hypergraph on the same set of vertices as $\mathcal{H}$ consisting of the fat hyperedges, then

Lemma 2. If $r=3$, then

$$
|E(\mathcal{H} \backslash \mathcal{F})| \leq \frac{(k-1) n}{2}
$$

If $r>3$, then

$$
|E(\mathcal{H} \backslash \mathcal{F})| \leq \frac{(k-1)^{2} n}{2}
$$

Proof. Arbitrarily select a thin edge from each $h \in \mathcal{H} \backslash \mathcal{F}$. Let $G$ be the graph consisting of the selected thin edges. We know that each edge in $G$ was selected at most once if $r=3$ and at most $k-1$ times in the $r>3$. Thus, we have that $|\mathcal{H} \backslash \mathcal{F}| \leq|E(G)|$ for $r=3$ and $|\mathcal{H} \backslash \mathcal{F}| \leq(k-1)|E(G)|$ for $r>3$. Moreover, $G$ is $P_{k}$-free since a $P_{k}$ in $G$ would imply a Berge $P_{k}$ in $\mathcal{H}$ by considering any hyperedge from which each edge was selected. It follows by Theorem 1 that $|E(G)| \leq \frac{(k-1) n}{2}$, so $|\mathcal{H} \backslash \mathcal{F}| \leq \frac{(k-1) n}{2}$ if $r=3$, and $|\mathcal{H} \backslash \mathcal{F}| \leq \frac{(k-1)^{2} n}{2}$ if $r>3$. 
Any hyperedge of $\mathcal{F}$ contains only fat edges, so it corresponds to a unique $r$-clique in $F$. This implies the following.

Observation 1. The number of hyperedges in $E(\mathcal{F})$ is at most the number of $r$-cliques in the fat graph $F$.

To this end we will upper bound the number of $r$-cliques in $F$, by making use of the following important lemma.

Lemma 3. There are no two disjoint cycles of length at least $k / 2+1$ in the fat graph $F$.

Proof. Let $C$ and $D$ be two such cycles. By connectivity, there are vertices $v \in V(C)$ and $w \in V(D)$ and a Berge path from $v$ to $w$ in $\mathcal{H}$ containing no additional vertices of $C$ or $D$ as defining vertices. This path can be extended using the hyperedges containing the edges of $C$ and $D$ to produce a Berge path of length $k$ in $\mathcal{H}$ (note that here we used that the edges of $C$ and $D$ are fat), a contradiction.

Assume that $F$ has connected components $C_{1}, C_{2}, \ldots, C_{t}$. Trivially,

$$
N_{r}(F)=\sum_{i=1}^{t} N_{r}\left(C_{i}\right)
$$

If $\left|V\left(C_{i}\right)\right| \leq k / 2$, then trivially

$$
N_{r}\left(C_{i}\right) \leq\left(\begin{array}{c}
\left|V\left(C_{i}\right)\right| \\
r
\end{array}\right) \leq \frac{\left|V\left(C_{i}\right)\right|^{r}}{r !} \leq \frac{k^{r-1}\left|V\left(C_{i}\right)\right|}{2^{r-1}(r-1) !} .
$$

So we can assume $\left|V\left(C_{i}\right)\right| \geq k / 2$. By Lemma 3, we have that for all but at most one $i, C_{i}$ does not contain a cycle of length at least $k / 2+1$. So by Corollary 1, for all but at most one $i$, say $i_{0}$, we have

$$
N_{r}\left(C_{i}\right) \leq \frac{\left|V\left(C_{i}\right)\right|-1}{k / 2-2}\left(\begin{array}{c}
k / 2-1 \\
r
\end{array}\right) \leq \frac{k^{r-1}\left|V\left(C_{i}\right)\right|}{2^{r-1}(r-1) !}+O\left(k^{r-2}\right) .
$$

If $\left|V\left(C_{i_{0}}\right)\right| \geq c_{k, r}$, then by Lemma 1 and by Corollary 2 we have

$$
N_{r}\left(C_{i_{0}}\right) \leq \frac{k^{r-1}\left|V\left(C_{i}\right)\right|}{2^{r-1}(r-1) !}
$$

Otherwise, $N_{r}\left(C_{i_{0}}\right) \leq\left(\begin{array}{c}\left|V\left(C_{i_{0}}\right)\right| \\ r\end{array}\right)=o(n)$. Therefore, by (11), we have

$$
\begin{gathered}
N_{r}(F)=\sum_{i=1}^{t} N_{r}\left(C_{i}\right) \leq \\
\leq \sum_{i=1}^{t}\left(\frac{k^{r-1}\left|V\left(C_{i}\right)\right|}{2^{r-1}(r-1) !}+O\left(k^{r-2}\right)\right)+o(n) \leq \frac{k^{r-1} n}{2^{r-1}(r-1) !}+O\left(k^{r-2}\right) n+o(n) .
\end{gathered}
$$


Therefore, by Observation 1,

$$
|E(\mathcal{F})| \leq N_{r}(F) \leq \frac{k^{r-1} n}{2^{r-1}(r-1) !}+O\left(k^{r-2}\right) n+o(n) .
$$

Since $|E(\mathcal{H})|=|E(\mathcal{H} \backslash \mathcal{F})|+|E(\mathcal{F})|$, adding up the upper bounds in (2) and Lemma 2, we obtain the desired upper bound on $|E(\mathcal{H})|$.

\section{Acknowledgements}

The authors Gyơri, Methuku, Salia and Tompkins were supported by the National Research, Development and Innovation Office - NKFIH under the grant K116769.

The author Vizer was supported by the Hungarian National Research, Development and Innovation Office - NKFIH under the grant SNN 116095.

\section{References}

[1] P. N. Balister, E. Gyốri, J. Lehel, and R. H. Schelp. Connected graphs without long paths. Discrete Mathematics, 308(19):4487-4494, 2008.

[2] A. Davoodi, E. Gyốri, A. Methuku, and C. Tompkins. An Erdős-Gallai type theorem for hypergraphs. arXiv preprint arXiv:1608.03241, 2016.

[3] P. Erdôs and T. Gallai. On maximal paths and circuits of graphs. Acta Mathematica Hungarica, 10(3-4):337-356, 1959.

[4] E. Gyôri, Gy. Y. Katona, and N. Lemons. Hypergraph extensions of the Erdôs-Gallai theorem. European Journal of Combinatorics, 58:238-246, 2016.

[5] G. N. Kopylov. Maximal paths and cycles in a graph. DOKLADY AKADEMII NAUK SSSR, 234(1):19-21, 1977.

[6] R. Luo. The maximum number of cliques in graphs without long cycles. arXiv preprint arXiv:1701.07472, 2017. 